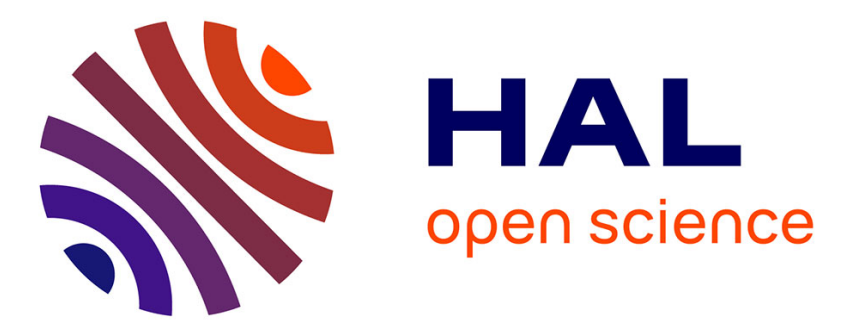

\title{
Comparison of image restoration methods for bioluminescence imaging
}

Smail Akkoul, Roger Lédée, Rémy Leconge, Christophe Léger, Rachid Harba, Sabrina Pesnel, Stéphanie Lerondel, Alain Lepape

\section{- To cite this version:}

Smaïl Akkoul, Roger Lédée, Rémy Leconge, Christophe Léger, Rachid Harba, et al.. Comparison of image restoration methods for bioluminescence imaging. ICISP 2008, Jul 2008, Cherbourg, France. pp.163-172. hal-00608075

\section{HAL Id: hal-00608075 https://hal.science/hal-00608075}

Submitted on 12 Jul 2011

HAL is a multi-disciplinary open access archive for the deposit and dissemination of scientific research documents, whether they are published or not. The documents may come from teaching and research institutions in France or abroad, or from public or private research centers.
L'archive ouverte pluridisciplinaire HAL, est destinée au dépôt et à la diffusion de documents scientifiques de niveau recherche, publiés ou non, émanant des établissements d'enseignement et de recherche français ou étrangers, des laboratoires publics ou privés. 


\title{
Comparison of Image Restoration Methods for Bioluminescence Imaging
}

\author{
Smaïl AKKOUL ${ }^{1}$, Roger LEDEE ${ }^{1}$, Remy LECONGE ${ }^{1}$, Christophe LEGER $^{1}$, Rachid HARBA \\ Sabrina PESNEL ${ }^{2}$, Stéphanie LERONDEL ${ }^{2}$, Alain LEPAPE ${ }^{2}$ \\ ${ }^{1}$ Université d'Orléans, Institut PRISME, 12 Rue de Blois, BP 6744, 45067 ORLEANS CEDEX 2 \\ ${ }^{2}$ Centre d'Imagerie du Petit Animal, CIPA-CNRS, UPR n ${ }^{\circ} 44,3$ B, Rue de la Férollerie, 45071 \\ Smail.Akkoul@univ-orleans.fr
}

\begin{abstract}
Bioluminescence imaging is a recent modality to visualize biological effects more especially for small animals. However the acquired images are degraded by diffusion and absorption phenomena from the tissue and by the acquisition system itself. In this paper, we use restoration methods to enhance the quality of bioluminescence images. We propose a model for image formation and an experimental determination of the PSF (Point Spread Function). Several methods of restoration are compared on test images generated according to the model and on real data. This comparison is insured by using MSE (Mean Square Error) and two quantitative criteria. Results establish that the statistical methods give more accurate restoration and well adapted for Bioluminescence Imaging.
\end{abstract}

Key Words: Restoration methods, Bioluminescence Imaging, acquisition models

\section{Introduction}

During the last years, research showed that there were numerous concordances between the human genome and that of primates, and mice [1]. With mice, the advantages are that we have a short generation time and convenient breeding conditions. It is possible to manipulate their human equivalent genes [2] to create models of human diseases [3]. But in vivo studies on these models need the development of novel methods of investigation adapted to small animals.

Among the emergent techniques for in-vivo investigation of the small animal, bioluminescence imaging (BLI) $[6,7]$ proves to be very promising. Bioluminescence is a chemical reaction which produces visible light that can be observed in a variety of marine and terrestrial animals, more especially in the firefly $[4,5]$. This imaging modality presents several advantages like its simplicity. It is non-invasive, low cost and it is at the same time, a functional and metabolic imaging of a gene expression [5]. Currently, it is exploited in complement of other methods [8], to monitor biological processes during time for tumors detection, drug therapy assessment and for follow-up of the neoplasic evolution $[2,3]$.

Nevertheless diffusion and absorption of light by the tissues [6] make this method difficult to use in practice, mainly when quantitative imaging is required. Moreover, the acquisition process introduces a point spread function which makes that acquired image is not matching to the reality [8]. One of the possible solutions to correct these effects would be to use image restoration methods to recover the original light sources [10]. As bioluminescence imaging is quite a new technique, no such work has been carried out to our knowledge.

In this study, the image restoration process is used to improve the quality and recover the spatial resolution of bioluminescence images. We investigate 
several methods of restoration and compare them on test images and real acquisitions.

This communication is organized as follow. In the second section, we present the material, the modeling of the system and all the methods of restoration studied as well as the parameters of comparison. The results obtained on synthesized images and real images are presented in third section. Conclusions of this work form the last section.

\section{Materiel and Methods}

\subsection{Acquisition System}

Bioluminescent imaging offers many opportunities for non-invasive study using reporter genes. Tagged cells are labeled with reporter genes encoding luciferase enzymes in living small animal. To monitor the evolution of tagged cells, substrate luciferin is applied to the animal by injection. Those cells that express the luciferase transgene emit photons. The mice were anesthetized, and placed onto warmed stage inside camera box. Our bioluminescent images were acquired for one minute with a commercial unit (Hamamatsu, Macroscopic Imaging AEQUORIA), that comprises, a light-tight cabinet with a high sensitivity cooled CCD camera. The images obtained have a resolution of $512 \times 512$ pixels coded on 16 bits and $300 \mu \mathrm{m}$ space resolution. An example of acquisition is presented in figure 1 .
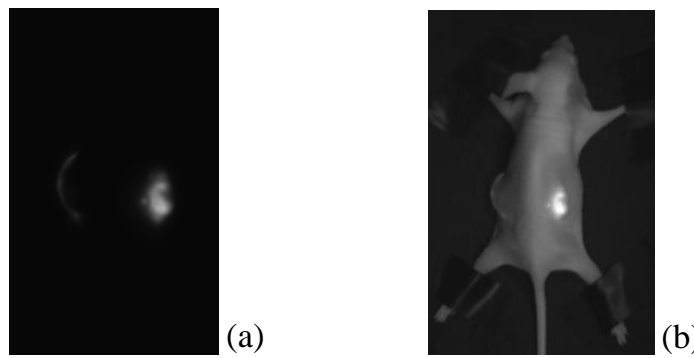

Fig. 1. (a) Example of a bioluminescence acquisition, (b) Fusion between of the bioluminescence image and visible light image.

\subsection{Acquisition Model}

The formation of the images of bioluminescence results from the counting of photons emitted by a source radiating in all directions starting from the inside of the small animal and which is collected outside by the CCD camera. The light intensity of the sources is low and photon propagation is subject to both scattering and absorption phenomena.

In this work, we will only consider subcutaneous tumors which represent $80 \%$ of tumor locations in experimental cancerology. So, we can measure the PSF including instrumentation and the skin effects. This is the first stage before including model of diffusion and absorption of the skin in the restoration process. Bioluminescence imaging acquisition process is similar to that of nuclear medicine, astronomical and optical microscopic imaging. All of them use CCD camera and a low light phenomena is recorded. The 
quantum nature of light leads to a Poisson modeling of the signal emitted by the object. Recent works on these imaging modalities provide reviews of proposed models $[20,23,25]$. The camera is cooled $\left(-75^{\circ} \mathrm{C}\right)$ to be extremely sensitive to the observed phenomena. Nevertheless, images are degraded by a noise due to CCD camera read-out, quantization, and dark current [9]. The additive noise is supposed to be a zero mean Gaussian noise of unknown variance $\sigma^{2}$. We propose to adopt for the bioluminescence imaging a Poisson model. This model is expressed as:

$$
i(x, y)=p([o(x, y) * h(x, y)])+b(x, y),
$$

where $i$ : is acquired image, $o$ : real image, $h$ : point spread function, *: convolution product, $p$ is the Poisson process, $b$ : the additive Gaussian noise.

\subsection{Restoration Methods}

The image restoration process is used to reduce the effects of the blur. This inverse problem has led to a large amount of work [10, 15, 16, 19, 21], the main difficulties being the determination of the PSF and the additive noise $[22,24]$. Many classifications are proposed in the literature [23, 25]. In our work we propose to classify used methods in four classes: linear, nonlinear, statistical, and blind methods.

The first class concerns the linear methods, like Wiener $[10,11]$ filter or that of Tikhonov-Miller [13, 23]. To decrease the sensitivity to noise of the inverse filter and its singularities, the Wiener filter minimizes the mean square error between the original image and the restored one. It is easy to implement, but it requires a good knowledge of the noise spectrum. It is expressed:

$$
M S E=E\left[\left(o(x, y)-\hat{o}(x, y)^{2}\right] .\right.
$$

The Tikhonov-Miller filter found when minimizing the Tikhonov functional, which is the square difference between the acquired image and blurred estimate of the original object regularized by a Tikhonov energy bound. It is expressed:

$$
\phi(O(x, y))=\operatorname{argmin}\|i(x, y)-(h * o)(x, y)\|^{2}+\lambda\|p * h\|^{2},
$$

where, the first term represents the fidelity to the data $\mathrm{I}(\mathrm{x}, \mathrm{y})$, and the second the smoothing applied to the restored image: $p$ is high pass filter, $\lambda$ is the regularization parameter and represents the trade-off between the fidelity to the data and the smoothness of the restored image and the original image. This method is simple to implement, but it is very sensitive to errors in the PSF data used for estimation, leading to ringing artifact in the solution.

The second class includes the nonlinear methods. To solve the difficulties occurring within this class, constraints were integrated in these linear methods. Historically, the Jason-Van Cittert's algorithm is the first constraint method. It is an iterative algorithm that converges quickly. But it is not very effective in the case of images having a low $S N R$. Thereafter, this algorithm was improved by introduction of other constraints like limiting the intensity of the signal for example. A nonlinear approach of Tikhonov-Miller algorithm was also proposed. The minimum of the Tikhonov function is reached iteratively by clipping the estimated negative intensities to zero after 
each iteration. Usually, a conjugate gradient (CG) method is used for the iterative search operation [13]. During iterations, the steepest descent in direction of minimum of the function is calculated. The solution is expressed as:

$$
O_{k+1}=\left\{\begin{array}{ccc}
O_{k}+s_{k} p_{k} & \text { if } & O_{k}+s_{k} p_{k} \geq 0 \\
0 & \text { otherwise }
\end{array},\right.
$$

where $\mathrm{s}_{k}$ is the size of the optimal step and $p_{\mathrm{k}}$ the conjugate gradient direction.

The improvement of this algorithm was the objective of many work but all are based on the assumption that the nature of the noise is Gaussian [13]. To take into account other physical model as Poisson process, statistical methods were proposed and are classified in the third class. Statistical methods are based on the Bayes's rule. Consisting to construct the conditional probability density relationship:

$$
P(O / I)=P(I / O) P(O) / P(I),
$$

where $P(O / I)$ denotes the probability density function $(p d f)$ of $O$ given $I, P$ $(O)$ denotes the $p d f$ of real image, and $P(I / O)$ denotes the a priori probability. The objective is to find an estimate of the original image which maximizes the posterior probability. The maximization of the right part of the equation gives a solution $O$. This is obtained by only the maximization of the density $P(I / O)$ over $O$ (likelihood solution (ML)) or by maximization of the product $P(I / O)$ $P(O)$ (maximum a posteriori solution (MAP)). In both cases $P(I)$ is considered as constant value which has not effect in the maximization process, and is ignored. In this class, the Lucy-Richardson algorithm [16, 18, 19] is an iterative technique used heavily for the restoration based on Bayes approach. It is widely used in the restoration of astronomical images [25].

Blind deconvolution methods represent the last class of algorithms. The performances of the preceding methods are based on a good knowledge of the PSF and also of the noise $[12,24]$. The blind methods are more proper when the PSF and noises in the image are both unknown. Many of these algorithms derived from probabilistic modes compute and use the same approach as constrained iteration algorithms, including the both estimation of the PSF and the original image in each iteration. In these methods, estimate PSF can be injected to increase the convergence of calculations.

In addition to these four classes, methods based on the wavelets seem to give good results. In this work, we tested only the methods present in the four classes.

\subsection{Performance Measurement}

To measure the performances of restorations methods we use two tests. First, the restored results are compared at each iteration to original image using MSE. The MSE measures the difference in energy between the two compared images. The MSE is given as:

$$
M S E=\frac{1}{M N} \sum_{i, j}\left|O_{i, j}-\hat{O}_{i, j}\right|^{2} .
$$

But this measure is global and sometime it is difficult to choose the best method between many approaches. So, in order to completely assess the 
success of our restoration procedures, we use a specific evaluation criteria based on that proposed in [14] and used by Mignotte and Meunier for SPECT images [17]. We define regions of interest (ROI) which are the objects in the test image with a high gray level. In these specific areas, we compute the mean of the gray levels to compare it to the grey level of the same ROI in the original image. Also, standard deviation of this mean is calculated. For a good restoration, the mean value must tend to the original value and the standard deviation must decreases towards zero.

\section{Experiments and Results}

\subsection{PSF of the System}

Since the application concerned the subcutaneous tumors, we record the PSF of the acquisition system including the skin of mouse in order to take into account for the phenomena of absorption and diffusion of this part of the animal. To do that, we realized an acquisition system which is composed by a point source obtained using optical fiber which diameter of the heart is equal to $200 \mu \mathrm{m}$, lower than the size of a pixel of the image (size of the pixel being of $300 \mu \mathrm{m}$ ). To remain in the spectral band of the luciferase in vivo, the optical fiber is supplied by a white light source filtering by two interferometric filters. Then, to reduce the effects of the noise, the acquisition was carried twenty times under the same conditions and the sum of acquisitions was divided by the number of acquisitions.

\subsection{Generation of Test Images}

To test the performance of the restoration algorithms, we generated two test images integrating the imperfections of the acquisition system. The images are realized as a convolution between the undistorted image of shapes and the experimental PSF. Then, Gaussian noise of the system is added to respect equation 1. The figure 4 represents the two test images before and after degradation. The first image is formed by a disc of ray $2.25 \mathrm{~mm}$ with a uniform intensity of $6.10^{4}$ and the second by two small close discs of ray 900 $\mu \mathrm{m}$ separated by $300 \mu \mathrm{m}$.
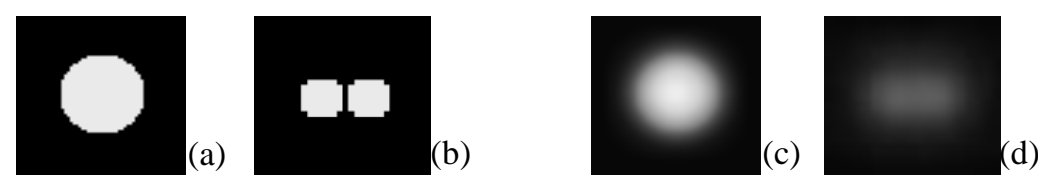

Fig.1 (a) (b) Test image before degradation (c) (d) Test image after degradation

\subsection{Restoration of the Test Images}

First, we have tested the deconvolution methods on test images. The advantages to work with synthetic degraded images are that we know exactly the initial object, and the degradation model. Then, it is easy to quantify results by comparing the restored image to the original image (figure 4a). To quantify the general quality of restoration, we compute the MSE between the restored image and initial undegraded image for each method and for each 
iteration. We report on the table 1 two objects after restoration with the minimum measure of the MSE. In first consideration, we observe that statistical methods and the Tikholov Miller method with a good choice of parameters give the best results. But the level of gray under the shapes presents ringing artifacts.

\begin{tabular}{|c|c|c|c|c|c|c|c|c|c|}
\hline \multirow{2}{*}{$\begin{array}{l}\text { Tested } \\
\text { Image }\end{array}$} & \multicolumn{2}{|c|}{ Linear Methods } & \multicolumn{3}{|c|}{ Nonlinear Methods } & \multicolumn{3}{|c|}{ Statistical Methods } & \begin{tabular}{|c|} 
Blind \\
Methods
\end{tabular} \\
\hline & $\mathrm{W}$ & TM & GM & SD & CG & LR & MAP & SMAR & ML \\
\hline & & & & & & & & & \\
\hline $\begin{array}{l}\text { MSE } \\
\left(10^{6}\right) \\
\end{array}$ & 7.312 & 4.797 & 5.830 & 6.008 & 15.634 & 4.514 & 4.5157 & 4.732 & 4.441 \\
\hline $\mathrm{N}^{\circ}$ Iterations & - & - & 20 & 20 & 250 & 250 & 250 & 250 & 250 \\
\hline
\end{tabular}

Tab.1. Restoration results of the test images by the different methods, with the minimal MSE. W: wiener, TM: Tikhonov Miller, GM: Golden Meinel, SD: Steepest Decent, CG: Conjugate Gradient, LR: Lucy Richardson, MAP: Maximum a posteriori, SMAR: Simultaneous Multiplicative Algebraïc Restoration, ML: maximum Liklhood.

The second experiment tests the ability of the methods to improve the quantitative measurement of the total amount of bioluminescence inside the disc area (ROI). The figures $5 \mathrm{a}$ and $5 \mathrm{~b}$ show the evolution of the two parameters as function of iteration number. From figure of mean we can see that this parameter for all methods converges to the real value $\left(610^{4}\right)$ except for gradient conjugate which over-estimates this value. For the standard deviation the statistical methods have small gap and continue to decrease with iterations. On the other hand the nonlinear methods diverge after some iterations. To obtain a quantitative comparison, we take the two parameters with their optimal values i.e. the value of the number of iterations corresponding to minimum of the MSE. This result is summarized in table2. With our criteria exposed in II.4, in the table 2 and with the first results (table 1 ), it is easily seen that statistical restoration methods give good results.
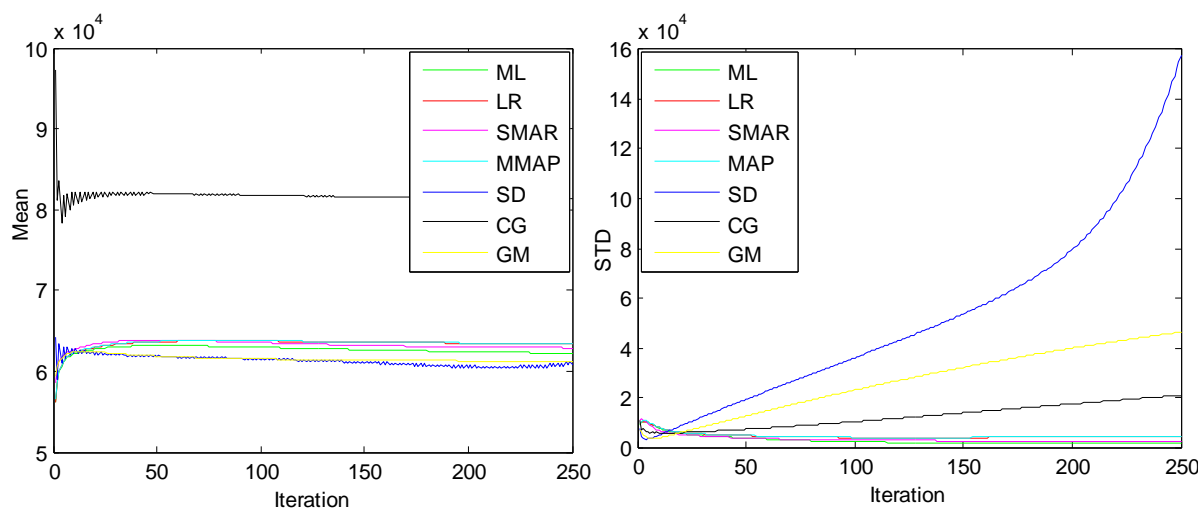

Fig.5. (a) Mean of the ROI at each iteration, (b) Standard deviation of ROI at each iteration. 


\begin{tabular}{|c|c|c|c|c|c|c|c|c|c|}
\hline \multirow{2}{*}{} & \multicolumn{2}{|l|}{ Linear Methods } & \multicolumn{3}{|c|}{ Nonlinear Methods } & \multicolumn{2}{|c|}{ Statistical Methods } & $\begin{array}{c}\text { Blind } \\
\text { Methods }\end{array}$ \\
\cline { 2 - 12 } & $\mathrm{W}$ & $\mathrm{TM}$ & $\mathrm{GM}$ & $\mathrm{SD}$ & $\mathrm{CG}$ & $\mathrm{LR}$ & MAP & SMAR & ML \\
& & & & & & & & & \\
\hline Mean $\left(10^{4}\right)$ & 6.167 & 6.207 & 6.239 & 6.207 & 8.133 & 6.335 & 6.337 & 6.289 & 6.223 \\
\hline STD $\left(10^{3}\right)$ & 15.322 & 7.279 & 5.936 & 8.604 & 22.066 & 4.361 & 4.383 & 2.508 & 1.558 \\
\hline $\mathrm{N}^{\circ}$ Iterations & - & - & 20 & 20 & 250 & 250 & 250 & 250 & 250 \\
\hline
\end{tabular}

Tab.2. Mean and standard deviation of the ROI after deconvolution for each method.

\subsection{Bioluminescence Images Restoration}

We have also tested the deconvolution algorithms on real bioluminescence images. We present two cases where the ROI of the real acquisition are reported in the table 2. The obtained results are showed in table 2 and we can see that statistical methods give restored images with limiting ringing artifacts and good spatial resolution. Note that it is difficult to define a quantitative measure of the deconvolution results because we do not know the original image.

\begin{tabular}{|c|c|c|c|c|c|c|c|c|c|}
\hline \multirow[b]{2}{*}{ Real image } & \multicolumn{2}{|c|}{ Linear Methods } & \multicolumn{3}{|c|}{ Nonlinear Methods } & \multicolumn{3}{|c|}{ Statistical Methods } & \multirow{2}{*}{\begin{tabular}{|c|}
$\begin{array}{c}\text { Blind } \\
\text { Methods }\end{array}$ \\
ML \\
\end{tabular}} \\
\hline & W & TM & GM & SD & CG & LR & MAP & SMAR & \\
\hline 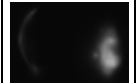 & & & ( & & & 8 & 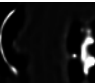 & 6 & 6 \\
\hline-8 & 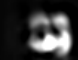 & 8 & 3 & (4) & & (3) & (3) & 3 & (3) \\
\hline
\end{tabular}

Tab.3. Restoration of real bioluminescence images with the same condition like tests images.

\section{Conclusions}

In this work nine algorithms for restoration methods were tested and compared on test images and on real bioluminescence images. To do this, we have defined a physical model of bioluminescence imaging formation. Then, we used an experimental approach to estimate more accuracy the point spread function of the acquisition system including the skin of mice. These allowed us to simulate synthetic data. After having defined and used comparison criteria, we conclude that statistical methods are more appropriated to bioluminescence imaging. On real data, we have noticed that the statistical restoration methods improve the contrast and spatial resolution of these images. To confirm this study, we will carry out physical measurements on small tumors and compare them to measures extracted from restored images.

\section{References}

1. J. A. Beck, S. Lloy, M. Hafezparast and al.: Genealogies of Mouse Inbred Strains. Nature Genetics 24, 23-25,(2000)

2. C. H. Contag, D. Jenkins, P.R Contag, R.S Negrin: Use of Reporter Genes for Optical Measurements of Neoplastic Disease In Vivo. Neoplasia, (2002), Vol. 2(1-2): 41-52 
3. M. Edinger, Y. Cao, and al: Advancing Animal Models of Neoplasia through in Vivo Bioluminescence Imaging. Vol. 38, European Journal of Cancer, Number 16, November (2002), pp. 2128-2136(9)

4. L. F. Greer, A. A. Szalay: Imaging of Light Emission from the Expression of Luciferases in Living Cells and Organisms: A Review. Vol. 17; Part 1, Luminescence -Chichester2002, P. 43-74

5. A. Roda, P. Pasini, M. Mirasoli, E. Michelini, M. Guardigli: Biotechnological Applications of Bioluminescence and Chemiluminescence. Vol. 22 No.6, Trends in Biotechnology, June (2004)

6. B. W. Rice, M. D. Cable, and M. B. Nelson: In Vivo Imaging of Light-Emitting Probes. Vol. 6, Iss 4, Journal of Biomedical Optics - October (2001) - pp. 432-440

7. R.T. Sadikot, T. S. Blackwell: Bioluminescence Imaging. Vol. 2, Proc Am Thorac Soc. pp 537-540, (2005)

8. A. Rehemtulla, L.D. Stegman, and al.: Rapid and Quantitative Assessment of Cancer Treatment Response Using In Vivo Bioluminescence Imaging. Vol. 2, Neoplasia, Number 6, 1 December (2000), pp. 491-495(5)

9. D. L. Snyder, A. M. Hammoud, and R. L. White: Image Recovery from Data Acquired with a Charge-Coupled-Device Camera. Vol. 10, J. of the Optical Society of America, pp. 1014-1023, May (1993)

10. M.R.Banham, A. Aggelos, K. Kastaggelos: Digital Image Restoration. IEEE, Signal Processing Magazine, 1053-5888/97.

11. A. Prakash Dhawan, and al.: Image Restoration by Wiener Deconvolution in Limited-View Computed Tomography. Vol. 24, December (1985), No. 23 / APPLIED OPTICS, Optical Society of America

12. D. Kundur, D. Hatzinakos: Blind Image Deconvolution Revisited. IEEE Transactions on Image Magazine, 1053-5888/96/(1996) IEEE

13. G.M.P. Van Kempen and al: A Quantitative Comparison of Image Restoration Methods for Confocal Microscopy. Vol. 185, Journal of Microscopy, Number 3, March 1997, pp. 354$365(12)$

14. S.Webb, A. Long, R. J. Ott, M. O. Leach, and M. A. Flower: Constrained Deconvolution of SPECT Liver Tomograms by Direct Digital Image Restoration. Vol. 12, Med. Phys. no.1, pp 53-58, (1985)

15. J. Biemond, R.L. Lagendijk, R.M. Merseau: Iterative Methods for Image Deblurring. 0018- 9219/90/0500-0856/(1990) IEEE.

16. W. H. Richardson: Bayesian-Based Iterative Method of Image Restoration. J. Optical Society of America. 62 (1), pp. 55 (1972)

17. M. Mignotte, J. Meunier: Three-Dimensional Blind Deconvolution of SPECT Images. Vol. 47, Iss 2, Biomedical Engineering, IEEE Transactions, Feb (2000) pp 274 - 280

18. L.A. Shepp and Y. Vardi, Maximum Likelihood reconstruction for emission tomography. IEEE Trans Med Imaging MI-1 (1982), pp. 113-122

19. Dempster, A. P., Laird, N. M. et Rubin, D. B: Maximum Likelihood from Incomplete Data Via the EM Algorithm (With Discussion). Journal of the Royal Statistical Society, B 39 :138. (1977)

20. H. Lantéri and C. Theys: Restoration of Astrophysical Images - The Case of Poisson Data with Additive Gaussian Noise. Vol. 2005, EURASIP Journal on Applied Signal Processing, Iss 15, pp. 2500-2513,(2005)

21. A.K. katsaggelo: Iterative Image restoration Algorithms. Vol. 28, Opt. Eng., No. 7, P. 735- 748/1989.

22. F. Rooms, et al.: PSF estimation with application in auto focus and image restoration, in: IEEE Signal Processing Symposium, Leuven, Belgium, March (2002)

23. P .Sarder, A. Nehorai: Deconvolution Methods for 3-D Fluorescence Microscopy Images. Vol. 23, Signal Processing Magazine, IEEE, No. 3. (2006), pp. 32-45.

24. H.Du and K. J. Voss: Effects of Point-Spread Function on Calibration and Radiometric Accuracy of CCD Camera. Optical Society of America, OCIS codes: 110.4100, 120.5630, $070.2590,100.3020,(2004)$

25. J.L. Starck, E. Pantin and F. Murtagh: Deconvolution in Astronomy: a Review. Vol. 114,Publication of the Astronomical Society of the Pacific. pp 1051-1069, (2002) 